\title{
4-(1H)-喹诺酮及甲氧基和溴代衍生物的合成研究
}

\author{
马梦林*,a,b 黄剑彪 ${ }^{a, c}$ 王玉良 ${ }^{b}$ 陈淑华 $b$ 陈 华 $b$ \\ $\left({ }^{a}\right.$ 西华大学物理与化学学院 成都 610039) \\ $\left({ }^{b}\right.$ 四川大学化学学院 教育部绿色化学重点实验室 成都 610064) \\ ( ${ }^{c}$ 上海大学理学院 上海 200444)
}

\begin{abstract}
摘要 提出了一种 4-(1H)-喹诺酮衍生物合成方法. 以 3,3-二乙氧基丙酸乙酯和苯胺为原料, 生成中间体 3-(苯基亚氨 基)丙酸乙酯，再经热环化或微波促进环化两步反应制备 4-(1H)-喹诺酮，并以此方法合成了 7 个溴代或甲氧基取代的 4-(1H)-喹诺酮衍生物.
\end{abstract}

关键词 4-(1H)-喹诺酮; 3,3-二乙氧基丙酸乙酯; 合成方法; 环化

\section{Syntheses of 4-(1H)-Quinolones and the Brominated or Methoxy Substituted Derivatives}

\author{
Ma, Menglin ${ }^{*, a, c}$ Huang, Jianbiao ${ }^{a, b} \quad$ Wang, Yuliang $^{c} \quad$ Chen, Shuhua $^{c} \quad$ Chen, Hua ${ }^{c}$ \\ ( ${ }^{a}$ School of Physics and Chemistry, Xihua University, Chengdu 610039) \\ ( ${ }^{b}$ Key Laboratory of Green Chemistry and Technology of Ministry of Education, Faculty of Chemistry, \\ Sichuan University, Chengdu 610064) \\ ( ${ }^{c}$ College of Science, Shanghai University, Shanghai 200444)
}

\begin{abstract}
A simple and new synthetic approach to 4-(1H)-quinolones was described. This method was started from ethyl 3,3-diethoxypropanoate and aniline via a synthetic sequence of preparation of key intermediate 3-(phenylimino)propionate and cyclization reaction. The brominated or methoxy substituted derivatives were synthesized by this new method as well.
\end{abstract}

Keywords 4(1H)quinolones, 3,3-diethoxypropanoate; synthetic method; cyclization

4-(1H)-喹诺酮及其衍生物被发现存在于许多天然 生物碱中, 具有广泛的生物活性 ${ }^{[1]}$. 4-(1H)-喹诺酮衍生 物是药物化学领域中常见的基础性活性结构单元, 如用 作抗疮虫活性组分治疗症疾 ${ }^{[2]}$ 、用作血小板衍生生长因 子(PDGF)受体抑制剂治疗心脑血管疾病 ${ }^{[3]}$ 、用作多巴胺 受体激动剂和铁螯合剂治疗帕金森氏病 ${ }^{[4]}$ 、用作亚型生 长抑素受体激动剂(SST2)治疗肢端肥大症和糖尿病性 视网膜病变 ${ }^{[5]}$ 以及用作 II 型拓扑异构酶细菌抑制剂 ${ }^{[6]}$ 等.

Gould-Jacobs 喹啉合成法是 4-(1H)-喹诺酮最为经典 的合成方法 ${ }^{[7]}$, 现仍被大量用于药物分子中 4-(1H)-㨒诺 酮结构单元的合成 ${ }^{[2,2 d, 3,3]}$. 其反应机理 ${ }^{[8]}($ Scheme 1) 为 芳胺与烷氧亚甲基丙二酸酯(2)缩合得到苯酰胺亚甲基 丙二酸酯(3), 再环化生成 4-羊基-3-烷氧羰基喹啉(5), 化合物 5 经水解、脱羧得 4-(1H)-喹诺酮衍生物. 由于环
化中间体 4 在 3-位及亚甲基酮碳上有吸电子基, 热环反 较容易, 能在 $200{ }^{\circ} \mathrm{C}$ 左右温度下实现关环, 但这个吸电 子基却保留在了反应产物上, 不得不进行水解和高温脱 羧, 相应增加了合成难度, 影响了收率.

目前常用改进的 Gould-Jacobs 反应合成药物分子中 的 4-(1H)-喹诺酮结构单元 ${ }^{[2,2,2 c, 4,6,9]}$, 如以氰基醋酸酯、 $\beta$ 酮酯或米氏酸(Meldrum acid)替换烷氧亚甲基丙二酸酯. 相应的机理 ${ }^{[8]}$ (Scheme 2)为芳胺与米氏酸(Meldrum acid) 6 反应生成相应的缩合产物 7, 化合物 7 通过闪式真空热 解法(flash vacuum pyrolysis conditions)脱丙酮和二氧化 碳, 形成一个亚甲基酮(a kumulenone species) 8, 经过 1,3- $\mathrm{N}-\mathrm{H}$ 移位, 再环化、氢转移生成 4-差基喹啉或类似 物. 不过，上述两条合成路线均存在一定的不足，国内 外学者提出了一些改进方法, 如以可循环的丙二酸酯树

* E-mail: mmlchem@163.com

Received October 17, 2012; revised November 5, 2012; published online November 15, 2012.

Project supported by the National Natural Science Foundation of China (No. B020601), the Natural Science Foundation of Sichuan Province (No. 07ZA109). 国家自然科学基金(No. B020601)、四川省自然科学重点基金(No. 07ZA109)资助项目. 
<smiles>CCOC(=O)CC(=O)OCC</smiles><smiles>CCOC(=O)C(=CNc1ccccc1)C(=O)OCC</smiles>

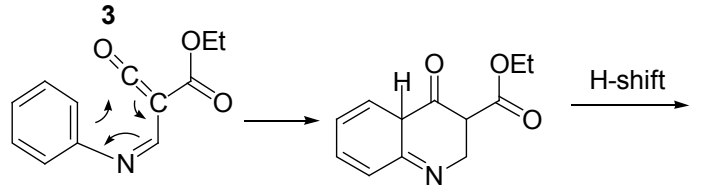

4<smiles>CCOC(=O)C1=CN(S)C2C=CC=CC2C(O)=C1C(=O)OCC</smiles><smiles>O=C(O)C1=C(O)C2C=CC=CC2N=C1</smiles>

Scheme 1<smiles>[R]OC1C(=O)OC(C)(C)OC1=COC</smiles><smiles>[R]c1cccc(NC=C2C(=O)OCOC2=O)c1NCc1ccccc1Nc1ccccc1</smiles>

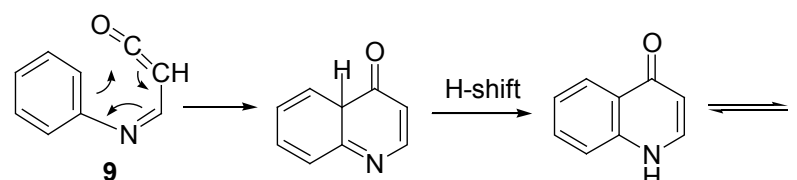<smiles>OC1=CC=NC2C=CC=CC12</smiles>

1a

Scheme 2

脂代替丙二酸酯的固相反应合成 4-(1H)-喹诺酮等 ${ }^{[10]}$.

本文用 3,3-二乙氧基丙酸乙酯(10)代替烷氧亚甲基 丙二酸酯(2)或米氏酸(6), 合成了 4-(1H)-喹诺酮和甲氧 基及溴代衍生物, 见 Scheme 3. 合成方法能有效避免上 述合成方法中最为繁琐、条件最为苛刻和收率低的步骤. 如，其一，能有效避免以烷氧亚甲基丙二酸酯为原料合 成路线中的水解、脱羧步骤; 其二，可省去在以米氏酸 烷为原料 Gould-Jacobs 反应中闪式真空热解法脱丙酮和
二氧化碳步骤. 以此两步 4-羟基喹啉合成方法合成了 8 个 6,7 或 8 位溴代或甲氧基取代的 4-差基喹啉衍生物 $\mathbf{1 a} \sim \mathbf{1 h}$.

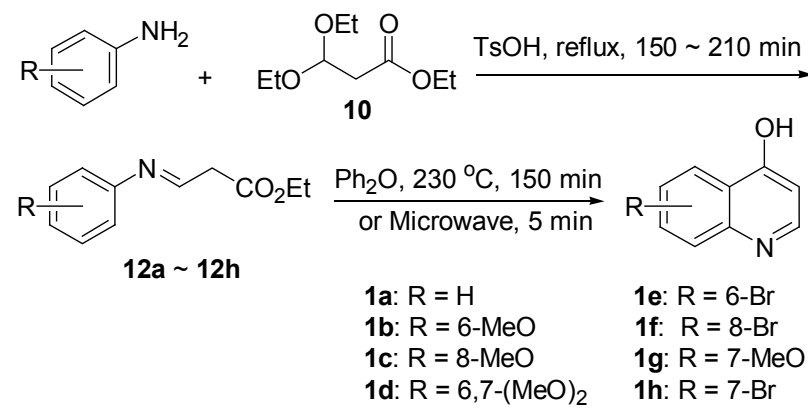

Scheme 3

\section{1 结果与讨论}

\section{1 热环环反应方法与时间考察}

热环化反应是成功合成目标物最关键的步骤，文献 中类似化合物的环化常使用高沸点溶剂[例如，二苯醚 $\left(\mathrm{Ph}_{2} \mathrm{O}\right)$ ，DOWTHERMTM，四甘醇二甲醚等]或无溶剂条 件下高温反应实现环化. 烷氧亚甲基丙二酸酯为原料的 合成路线中，中间体 $\mathbf{3}$ 的环化温度多在 $200{ }^{\circ} \mathrm{C}$, 而本文 中间体 3-(取代苯基亚氨基)丙酸乙酯(12)在 $200{ }^{\circ} \mathrm{C}$ 时几 乎不反应, 升高温度至 $230{ }^{\circ} \mathrm{C}$ 后才能实现环化, 获得目 标产物. 分析认为中间体 12 与 3 相比，在 3-位及亚甲基 酮碳上无吸电子基, 环化相对困难, 温度要求也较高. 另与米氏酸为原料的中间体 7 环化所需要的 $260{ }^{\circ} \mathrm{C}$ 甚 至更高的温度相比，中间体 $\mathbf{1 2}$ 的环化温度又低得多，应 为中间体 12 脱乙醇比中间体 7 脱丙酮和二氧化碳容易 所致.

Gould-Jacobs 反应热环化要求尽量短的反应时间, 以避免副反应，但短的反应时间会导致反应不完全，收 率偏低, 本文以化合物 $1 \mathrm{a}$ 的合成考察了热环化反应时 间对反应的收率的影响，反应时间 $150 \mathrm{~min}$ 时获得了最 好的收率 87\%(表 1, Entry 4).

微波促进有机反应已有非常多成功实例, 特别是在 此类高温反应中微波促进反应的效果更为明显 ${ }^{[11]}$. 尝 试以微波代替传统加热方式实现 3-(苯基亚氨基)丙酸乙 酯 12 的热环化，大大的缩短了反应时间，收率也有一定 地提高(表 1, Entry 3).

\section{2 反应机理讨论}

以 3,3-二乙氧基丙酸乙酯(10)为原料的 4-(1H)-喹诺 酮衍生物的合成机理分析如 Scheme 4, 中间体 12a $12 \mathrm{~h}$ 在高温或微波环境下脱醇，形成一个亚甲基酮 9a 9h, 其经环化、氢转移生成 4-羞基基喹啉或类似物. 从机 理看，过渡态 $9 \mathrm{a} \sim 9 \mathrm{~h}$ 的亚甲酮碳上没有吸电子基，与连 
表 1 环化合成 $\mathbf{1 a} \sim \mathbf{1 h}$ 反应时间与收率

Table 1 Reaction time and yield of synthesis of compounds $\mathbf{1 a} \sim \mathbf{1 h}$

\begin{tabular}{|c|c|c|c|c|c|}
\hline \multirow{2}{*}{ Entry } & \multirow{2}{*}{ Compd. } & \multicolumn{2}{|c|}{ Thermal ring closure } & \multicolumn{2}{|c|}{ Microsave promoted closure } \\
\hline & & Reaction time/min & Yield $^{a} / \%$ & Reaction time/min & Yield $^{a} / \%$ \\
\hline 1 & & 60 & 21 & & \\
\hline 2 & & 90 & 33 & 3 & 56 \\
\hline 3 & $\mathbf{1 a}$ & 120 & 75 & 5 & 89 \\
\hline 4 & & 150 & 87 & 10 & 21 \\
\hline 5 & & 180 & 69 & 15 & 13 \\
\hline 6 & $1 \mathrm{~b}$ & 150 & 89 & 5 & 89 \\
\hline 7 & $1 \mathrm{c}$ & 150 & 94 & 5 & 95 \\
\hline 8 & 1d & 150 & 98 & 5 & 98 \\
\hline 9 & 1e & 150 & 72 & 5 & 73 \\
\hline 10 & 1f & 150 & 88 & 5 & 88 \\
\hline 11 & $1 \mathrm{~g}^{b}$ & 150 & 60 & 5 & 69 \\
\hline 12 & $\mathbf{1} \mathbf{h}^{b}$ & 150 & 52 & 5 & 54 \\
\hline
\end{tabular}

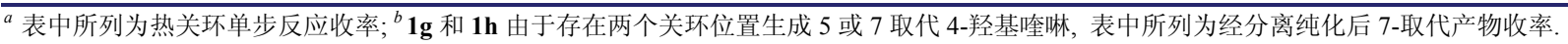<smiles>[R]COC(=O)CC(OCC)OCC</smiles>

$12 \mathrm{a} \sim 12 \mathrm{~h}$

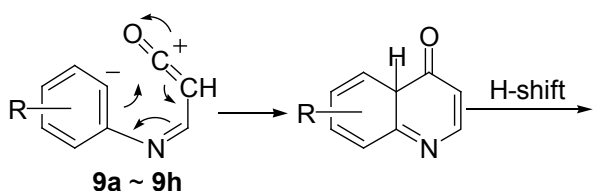

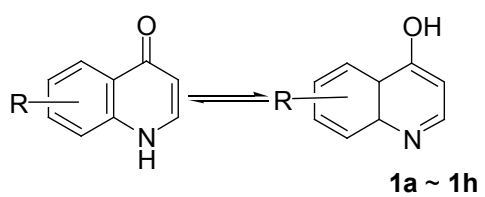

Scheme 4

有吸电子基的过渡态 $\mathbf{4}$ 相比, 环化需要在更高的温度条 件下才能完成, 这与实验结果一致.

根据机理分析，当环化时苯环这边有供电子基时， 能提供更有利的亲核关环的位置, 环化反应将变得相对 容易, 实验显示苯环上有供电子基的 $\mathbf{1 b}$ (表 1, Entry 6) 和 $\mathbf{1} \mathbf{c}$ (表 1, Entry 7)特别是有两个供电子基的 $\mathbf{1 d}$ (表 1, Entry 8)收率略高于不含供电子基的 $\mathbf{1 a}$ (表 1, Entry 4).

\section{3 环化反应区域选择性}

与传统烷氧亚甲基丙二酸酯(2)或米氏酸 6 为原料 的 Gould-Jacobs 关环一样, 区域选择性同样是一个重要 的问题, 受空间位置和电子效应的影响可能导致混合产 物的生成. 如 Scheme 5 所示, 中间体 12b, 12c, 12e 和 $12 \mathrm{f}$ 其环化位置单一, 因此只有相应的 6 或 8 位取代目 标物生成.

中间体 $12 \mathrm{~g}$ 和 $12 \mathrm{~h}$, 其存在两个亲核关环的反应位 置, 得到 5 或 7 取代 4-差基喹啉混合物. 在 GouldJacobs $^{[8]}$, Skraup ${ }^{[12]}$ 和 Combes ${ }^{[13]}$ 等喹啉合成法中, 给电<smiles>[R]c1ccccc1N=CCC(OC)OCC</smiles>

12b $\mathrm{R}=\mathrm{MeO}$ $12 \mathrm{e} R=\mathrm{Br}$<smiles>[R20][R20](=O)Cc1c([R])ccc(I)c1/N=C\CC(=O)OCC</smiles><smiles>[R]c1cc(C)c(/N=C\CC(=O)OCC)c(I)c1</smiles>
$12 \mathrm{c} R=\mathrm{MeO}$ $12 f \mathrm{R}=\mathrm{Br}$<smiles>CCOC(=O)C/C=N\c1ccc(OC)c(OC)c1</smiles>

\section{Scheme 5}

子性基团均有利于对位环化产物的生成, 给电子能力越 强对位产物比例越高, 给电子能力强的甲氧基取代喹啉 7 位关环比应高于溴代产物, 与实验结果一致. 12d 由于 两个强供电子基的存在, 使得其只有一个关环产物, 6,7-二甲氧取代在其它喹啉合成法中均表现出了高的区 域选择性 ${ }^{[3 a]}$.

\section{2 结论}

本文研究了以 3,3-二乙氧基丙酸乙酯为原料两步法 合成 4-(1H)-喹诺酮衍生物的新合成路线, 考察了热环 化与微波促进环化两种方法的反应时间、温度等条件, 并以此方法制备了 8 个 6,7 或 8 位溴代或甲氧基取代的 4-羞弪基喹啉衍生物.

\section{3 实验部分}

\section{1 仪器与试剂}

MS 由 Finnigan-MAT 4510 型质谱仪测定(EI); ${ }^{1} \mathrm{H}$ NMR 由 Varian INOVA400 型核磁共振仪测定(DMSO- $d_{6}$ 
作溶剂, TMS 作内标); HPLC 由岛津 LC-20 高效液相色 谱仪测定 $\left(\mathrm{C}_{18}\right.$ 柱, $254 \mathrm{~nm}$, 流动相 $V$ (甲醇) : $V($ 水 $)=$ $90 ： 10$, 面积归一化法); 微波反应仪器为上海新仪微 波化学科技有限公司 MWave-5000多功能微波化学反应 仪.

3,3-二乙氧基丙酸乙酯阿拉丁试剂; 苯胺及取代苯 胺, $\mathrm{Ph}_{2} \mathrm{O}, \mathrm{TsOH}$ 成都长钲试剂; 溶剂等为市售分析纯试 剂; 薄层板: 硅胶 GF254(青岛海洋化工厂产品), $0.6 \%$ 的羧甲基纤维素钠铺板, $110{ }^{\circ} \mathrm{C}$ 活化 $1 \mathrm{~h}$.

\section{2 中间体 3-(取代苯基亚氨基)丙酸乙酯 12a 12h 的 合成}

向一 $50 \mathrm{~mL}$ 三颈瓶中加入 $24.7 \mathrm{mmol}$ 取代苯胺和 $4.75 \mathrm{~g}(24.7 \mathrm{mmol})$ 的 3,3-二乙氧基丙酸乙酯, 再加入 0.1 $\mathrm{g}(1.92 \mathrm{mmol})$ 的 $\mathrm{TsOH}$, 将反应体系加热至 $93{ }^{\circ} \mathrm{C}$ 反应 150 210 min; TLC 监测反应 ( $V$ (石油醚)： $V$ (乙酸乙 酯) $=4: 1$, 反应毕, 冷却反应体系, 反应混合物以石 油醚为洗脱剂柱色谱分离, 得中间体 $12 \mathrm{a} \sim 12 \mathrm{~h}$, 收率 $63 \% \sim 85 \%$.

\section{3 目标物 $1 \mathrm{a} \sim 1 \mathrm{~h}$ 的环化方法}

热环化: 将 $13.08 \mathrm{mmol} \mathbf{1 2 a} \sim \mathbf{1 2 h}$ 投入 $30 \mathrm{~mL}$ $\mathrm{Ph}_{2} \mathrm{O}$ 中, 慢慢加热到 $230{ }^{\circ} \mathrm{C}$ 反应 $150 \mathrm{~min}, \mathrm{TLC}$ [ $V$ (石 油醚) $: V($ 丙酮 $)=1: 2$ ]监测反应毕, 冷却至室温有 固体析出, 过滤得 $\mathbf{1 a} \sim \mathbf{1 h}$ 粗品; 微波促进环化: 将 $13.08 \mathrm{mmol} \mathrm{12a} \sim 12 \mathrm{~h}$ 投入 $30 \mathrm{~mL} \mathrm{Ph}_{2} \mathrm{O}$ 中，微波辐射 $5 \mathrm{~min}$, 冷却至室温有固体析出, 过滤得 $\mathbf{1 a} \sim \mathbf{1 h}$ 粗品.

\section{4 目标物 $1 \mathrm{a} \sim 1 \mathrm{~h}$ 的纯化方法与结构表征}

向上述 1a～1f 粗品中加入 $30 \mathrm{~mL}$ 石油醚，室温下充 分搅拌 $30 \mathrm{~min}$, 过滤, 再重复洗涤 2 次, 过滤后真空干 燥得 $\mathbf{1 a} \sim 1 \mathbf{f}$ 纯品.

4-(1H)- 喹诺酮 (1a) ${ }^{[9 b]}$ : ${ }^{1} \mathrm{H}$ NMR (DMSO- $d_{6}, 400$ MHz) $\delta$ : $6.54(\mathrm{~d}, J=9.0 \mathrm{~Hz}, 1 \mathrm{H}), 7.38(\mathrm{dd}, J=8.0,3.0 \mathrm{~Hz}$, $1 \mathrm{H}), 7.59(\mathrm{~d}, J=8.0 \mathrm{~Hz}, 1 \mathrm{H}), 7.75(\mathrm{~d}, J=8.0 \mathrm{~Hz}, 1 \mathrm{H}), 8.14$ (d, $J=8.0 \mathrm{~Hz}, 1 \mathrm{H}), 8.31$ (d, $J=9.0 \mathrm{~Hz}, 1 \mathrm{H})$; MS (ESI) $m / z$ : $146\left(\mathrm{M}^{+}+1\right)$.

6-甲氧基-4-(1H)-喹诺酮(1b) ${ }^{[14]}$ : ${ }^{1} \mathrm{H}$ NMR (DMSO$\left.d_{6}, 400 \mathrm{MHz}\right) \delta: 3.81\left(\mathrm{~s}, 3 \mathrm{H}, \mathrm{OCH}_{3}\right), 6.00(\mathrm{~d}, J=3.0 \mathrm{~Hz}$, $1 \mathrm{H}), 7.29(\mathrm{~d}, J=8.0 \mathrm{~Hz}, 1 \mathrm{H}), 7.52 \sim 7.49(\mathrm{~m}, 2 \mathrm{H}), 7.83$ (dd, $J=3.0,8.0 \mathrm{~Hz}, 1 \mathrm{H}), 11.93$ (s, $1 \mathrm{H}, \mathrm{OH})$; MS (ESI) $m / z$ : $176\left(\mathrm{M}^{+}+1\right)$.

8-甲氧基-4-(1H)-喹诺酮(1c) ${ }^{[4]}:{ }^{1} \mathrm{H}$ NMR (DMSO- $d_{6}$, $400 \mathrm{MHz}) \delta: 4.11\left(\mathrm{~s}, 3 \mathrm{H}, \mathrm{OCH}_{3}\right), 7.04(\mathrm{~d}, J=8.0 \mathrm{~Hz}, 1 \mathrm{H})$, $7.48 \sim 7.44(\mathrm{~m}, 2 \mathrm{H}), 7.60(\mathrm{~d}, J=8.0 \mathrm{~Hz}, 1 \mathrm{H}), 7.92(\mathrm{~d}, J=$ $3.0 \mathrm{~Hz}, 1 \mathrm{H})$; MS (ESI) $m / z: 176\left(\mathrm{M}^{+}+1\right)$.

6,7-二甲氧基-4-(1H)-喹诺酮 (1d $)^{[3 \mathrm{a}]}$ : ${ }^{1} \mathrm{H} \quad \mathrm{NMR}$
(DMSO- $\left.d_{6}, 400 \mathrm{MHz}\right) \delta: 3.82\left(\mathrm{~s}, 3 \mathrm{H}, \mathrm{OCH}_{3}\right), 3.86(\mathrm{~s}, 3 \mathrm{H}$, $\left.\mathrm{OCH}_{3}\right), 5.94(\mathrm{~d}, J=6.0 \mathrm{~Hz}, 1 \mathrm{H}), 6.70(\mathrm{~s}, 1 \mathrm{H}), 7.44(\mathrm{~s}, 1 \mathrm{H})$, 7.80 (d, $J=6.0 \mathrm{~Hz}, 1 \mathrm{H}), 8.15$ (s, 1H); MS (ESI) $\mathrm{m} / z: 206$ $\left(\mathrm{M}^{+}+1\right)$.

6-溴-4-(1H)-喹诺酮(1e) ${ }^{[15]}:{ }^{1} \mathrm{H}$ NMR (DMSO- $d_{6}, 400$ MHz) $\delta: 6.24(\mathrm{~d}, J=8.0 \mathrm{~Hz}, 1 \mathrm{H}), 7.50(\mathrm{dd}, J=8.0,2.4 \mathrm{~Hz}$, 1H), 7.97 (d, $J=8.0 \mathrm{~Hz}, 1 \mathrm{H}, 1 \mathrm{H}), 8.16(\mathrm{~d}, J=8.0 \mathrm{~Hz}, 1 \mathrm{H}$, $1 \mathrm{H}), 8.25$ (d, $J=9.0 \mathrm{~Hz}, 1 \mathrm{H}, 1 \mathrm{H}), 11.85(\mathrm{~s}, 1 \mathrm{H}, \mathrm{OH}) ; \mathrm{MS}$ (ESI) $m / z: 224\left(\mathrm{M}^{+}+1\right), 226\left(\mathrm{M}^{+}+3\right)$.

8-澳-4-(1H)-喹诺酮(1f) ${ }^{[15]}:{ }^{1} \mathrm{H}$ NMR (DMSO- $d_{6}, 400$ MHz) $\delta: 6.13(\mathrm{~d}, J=8.0 \mathrm{~Hz}, 1 \mathrm{H}), 7.24(\mathrm{dd}, J=8.0,3.0 \mathrm{~Hz}$, $1 \mathrm{H}), 7.86(\mathrm{~s}, 1 \mathrm{H}), 7.97(\mathrm{~d}, J=8.0 \mathrm{~Hz}, 1 \mathrm{H}, 1 \mathrm{H}), 8.11(\mathrm{~d}$, $J=9.0 \mathrm{~Hz}, 1 \mathrm{H}, 1 \mathrm{H}), 11.81$ (s, 1H, OH); MS (ESI) $m / z: 224$ $\left(\mathrm{M}^{+}+1\right), 226\left(\mathrm{M}^{+}+3\right)$.

上述 $1 \mathbf{g}$ 和 $1 \mathbf{h}$ 粗品加入 $30 \mathrm{~mL}$ 石油醚, 室温下充分 搅拌 $30 \mathrm{~min}$, 过滤, 再重复洗涤 2 次, 过滤后真空干燥 得得 5-和 7-位关环混合产物, HPLC 检测结果: 4-羟基-5甲氧基喹啉：4-差基-7-甲氧基喹啉 $(\mathbf{1 g})=18.2 ： 81.8 ; 4$ 羟基-5-澳喹啉：4-着基-7-澳喹啉 $(\mathbf{1 h})=25.4 ： 74.6$; 以 $30 \mathrm{~mL}$ 无水乙醇溶解粗品, 通入干燥 $\mathrm{HCl}$ 气体 $180 \mathrm{~min}$ 后过滤, 固体以 $10 \mathrm{~mL}$ 无水乙醇重结晶得喹啉盐酸盐纯 品, 氨水解离, 二氯甲烷萃取, 无水硫酸镁干燥, 蒸去 溶剂得 $1 \mathrm{~g}$ 和 $1 \mathrm{~h}$.

7-甲氧基-4-(1H)-喹诺酮 (1g) ${ }^{[16]}$ : ${ }^{1} \mathrm{H}$ NMR (DMSO$\left.d_{6}\right) \delta: 3.85\left(\mathrm{~s}, 3 \mathrm{H}, \mathrm{OCH}_{3}\right), 5.99(\mathrm{~d}, J=9.0 \mathrm{~Hz}, 1 \mathrm{H}), 6.91(\mathrm{~s}$, 1H), 6.93 (s, 1H), 7.85 (d, $J=9.0 \mathrm{~Hz}, 1 \mathrm{H}), 8.00$ (d, $J=8.0$ $\mathrm{Hz}, 1 \mathrm{H}), 11.65(1 \mathrm{H}, \mathrm{OH})$; MS (ESI) $m / z: 176\left(\mathrm{M}^{+}+1\right)$.

7-澳-4-(1H)-喹诺酮(1h) ${ }^{[17]}:{ }^{1} \mathrm{H}$ NMR (DMSO- $\left.d_{6}\right) \delta$ : $6.06(\mathrm{~d}, J=9.0 \mathrm{~Hz}, 1 \mathrm{H}), 7.46(\mathrm{~d}, J=9.0 \mathrm{~Hz}, 1 \mathrm{H}), 7.80(\mathrm{~s}$, $1 \mathrm{H}), 7.92(\mathrm{~d}, J=8.0 \mathrm{~Hz}, 1 \mathrm{H}), 8.00(\mathrm{~d}, J=8.0 \mathrm{~Hz}, 1 \mathrm{H})$, $12.01(\mathrm{~s}, 1 \mathrm{H}, \mathrm{OH}) ; \mathrm{MS}(\mathrm{ESI}) m / z: 224\left(\mathrm{M}^{+}+1\right), 226$ $\left(\mathrm{M}^{+}+3\right)$.

\section{References}

[1] Reitsema, R. H. Chem. Rev. 1948, 43, 43.

[2] (a) Jong, Y. H.; Takashi, K.; David, J. L.; Julie, A. C.; Kiplin, G. J. Med. Chem. 2011, 54, 7084.

(b) Dibyendu, D.; Frances, M. K.; Larry, D. B.; Donald, J. K. J. Med. Chem. 1998, 41, 4918.

(c) Peter, B. M.; John, S.; Ally P. L.; Kiplin, G. Bioorg. Med. Chem. Lett. 2005, 15, 1015.

(d) Hiroshi, S.; Nagwa, S. M.; Yusuke, W. Chem. Pharm. Bull. 2007, 55(5), 821

[3] (a) Takayuki, F.; Teruyuki, S.; Terufumi, S.; Tatsushi, O. J. Med. Chem. 2006, 49, 2186.

(b) Kazuo, K.; Ohyama, S. I.; Toshiyuki, S.; Atsuya T. Bioorg. Med. Chem. Lett. 2003, 11, 5117.

[4] Balaram, G.; Tamara, A.; Maarten, E. A. R.; Aloke, K. D. J. Med. Chem. 2010, 53, 2114.

[5] Nolt, M. B.; Zhao, Z. J.; Wolkenberg, S. E. Tetrahedron Lett. 2008, 
49, 3137.

[6] Laurent, G.; Michael, D. H.; Wu, J. J.; John, J. M. Bioorg. Med. Chem. Lett. 2007, 17, 2723.

[7] (a) Cilirles, C. P.; Ruystun, M. K. J. Am. Chem. Soc. 1946, 68, 1204.

(b) Byron, R.; Gerald, R. L.; Bernard, H. A. J. Am. Chem. Soc. 1946, 68, 1264.

[8] László, L.; Tibor, Z. N.; Gellért, S.; Richard, J.; György, D. Tetra. Lett. 2012, 53, 738.

[9] (a) Brandon, J. M.; Kimberly, A. L.; Dana, L. T. J. Org. Chem. 2007, 72, 2232.

(b) Raul, C.; Ricardo, T.; Valderrama, J. A. Synth. Commun. 1985, $15(2), 125$.

[10] Huang, X.; Liu, Z. X. Tetrahedron Lett. 2001, 42, 7655.

[11] Wang, J.; Jiang, F. C. Chin. J. Org. Chem. 2002, 22, 135 (in Chinese).
(王静，姜风超，有机化学，2002，22，135.)

[12] (a) Badger, G. M.; Crocker, H. P.; Ennis, B. C. Aust. J. Chem. 1963, 16, 814, 840

(b) Badger, G. M.; Ennis, B. C.; Matthews, W. E. Aust. J. Chem. 1963, 16, 828 .

[13] Bergstroem, F. W. Chem. Rev. 1944, 35, 156.

[14] Sabine, P.; Glenn, D. WO 2006021448, 2006 [Chem. Abstr. 2006 144, 274309].

[15] Duffy, K. J.; Chai, D. P.; Colon, M.; Fitch, D. M.; King, S. R.; Shaw, A. N.; Tedesco, R.; Wiggall, K. WO 2006132739, 2006 [Chem. Abstr. 2006, 146, 62743].

[16] Breault, G.; Eyermann, C. J.; Geng, B.; Morningstar, M.; Reck, F. WO 2006134378, 2006 [Chem. Abstr. 2006, 146, 81779].

[17] Prince, R. B.; Merrill, B. A.; Kshirsagar, T. A.; Heppner, P. D. WO 2006093514, 2006 [Chem. Abstr. 2006, 145, 314981].

(Li, L.; Lu, Z.) 